\title{
AN OBSTACLE DETECTION SYSTEM BASED ON MONOCULAR VISION FOR APPLE ORCHARD ROBOT
}

\author{
Jianfeng Zhang, ${ }^{*}$ Bin Yang, ${ }^{*}$ Nan Geng, ${ }^{*}$ and Lvwen Huang*
}

\begin{abstract}
Obstacle detection is one of the key problems in the autonomous movement of intelligent mobile robot. This paper focuses on the study of obstacle detection in the complex apple orchard. First, using $L^{*} a^{*} b^{*}$ (colour space for non-self-illuminated, named by CIE $1976 L^{*} a^{*} b^{*}$ or CIELAB) colour model to represent the apple orchard image, the obstacles in the robot's walking area can be effectively segmented from the complex background by onedimensional fuzzy entropy image segmentation algorithm. Then, the coordinate of the point close to the robot of the obstacle is obtained. Finally, the distance between the robot and the obstacle is estimated using the inverse perspective transformation. The experimental results show that the maximum absolute error is $7.87 \mathrm{~cm}$ and the maximum relative error is $6.53 \%$. The results can meet the accuracy requirements of the intelligent mobile application for an apple orchard robot.
\end{abstract}

\section{Key Words}

Apple orchard robot, obstacle detection, monocular vision, inverse perspective transformation

\section{Introduction}

Robot technology has been successfully applied in many fields, and it is more suitable for labour shortage due to its low cost, high precision and good reliability. In particular, robots can work in some dirty, dangerous and demeaning application area [1]. With the rapid development of robot technology and its successful application in the fields of industry [2], [3], it has become one of the hotspots in the research of intelligent agricultural robot [4]. Apple cultivation has the characteristics of large amount of labour, high precision operation and highly reproducible task, so it has a widely future application to develop the apple working robot which is suitable for modern apple orchard.

Obstacle positioning is one of the important problems of the autonomous movement for the intelligent mobile

* College of Information Engineering, Northwest A\&F University, Yangling 712100, China; e-mail: zjf@nwsuaf.edu.cn, \{b_yang, nangeng\}@nwsuaf.edu.cn, springchilly@gmail.com

Recommended by Prof. Chaomin Luo

(DOI: 10.2316/Journal.206.2017.6.206-5036) robot. To meet the requirements of the dynamic and unknown environment, the robot must be able to sense the changing environment, and independently and efficiently plan the moving path to avoid any obstacles. For autonomous walking in unknown and dynamic apple orchard environment, the robot mainly depends on the sensing system to know the environment and the robot's status information, which is one of the bases of the precise positioning, path planning and navigation control. Vision system is an important part of the mobile robot sensing system, which can obtain the robot working environment image through the visual sensor, and then, execute the image processing and recognition to realize the robot positioning and the moving area recognition. Traditional mobile robot vision system used panoramic vision, binocular vision, monocular vision combined with laser or ultrasonic ranging in most cases [5]-[7]. Based on binocular vision, Zhang et al. [8] obtained the 3D information of the obstacle by the object extraction algorithm of analysing the distribution of pixels in the scan line and realized the detection of the field obstacles. Based on monocular vision and ultrasonic ranging, Li et al. realized the fruit identification and localization of apple-picking robot [9]. Jia et al. [10] obtained the distance information of the road surface, crops and obstacles through the laser range device, which achieved the road edge detection of greenhouse robot. Although the machine vision in the scene detection, recognition and location of the obstacle has been given widespread attention, and it has also made some achievements, there are some shortcomings, such as the missing proximal information and high distance information noise for the panoramic vision. Meanwhile, there are some problems as the binocular vision, such as complex structure, high cost and complicated algorithms.

This paper presents a novel real-time, low cost and complexity method of obstacle detection based on monocular vision combining with the characteristics of the modern apple orchard and provides the basis for the autonomous motion of the robot. First, we analyse the characteristics of apple orchard image and use the $L^{*} a^{*} b^{*}$ colour model to represent it. Then, we segment the image by using one-dimensional fuzzy entropy image segmentation algorithm and remove residues, so as to obtain the coordinate 
of the point close to the robot of the obstacle. Finally, the ground coordinate of the obstacle is obtained by inverse perspective transformation, and thus the distance between the robot and the obstacle can be estimated.

\section{Analysis and Processing of Apple Orchard Image}

The modern apple orchard is a complex and semistructured environment. We should fully study the characteristics of the apple orchard image and select a reasonable image processing solution to ensure the effective identification of the robot's walking regions.

\subsection{Features of Apple Orchard Image}

Because the image acquisition environment cannot be strictly controlled in the field environment, the collected images have some problems, such as violent illumination variations, uneven exposure, shadow, foliage obscured and various colour texture. The problems are mainly expressed in the following:

(i) While the robot is walking, there are some changing factors, such as the weather, working time, traveling direction and others. These can cause more violent illumination variations shown as Fig. 1(a) and (b).

(ii) Because of different vegetation between two rows of apple trees, the image colour and texture features would be significantly different shown as Fig. 1(b), (c), (e) and (f).

(iii) The pavement also has different texture, such as grass pavement (Fig. 1(a)), little-cultivated soil pavement
(Fig. 1(c)), part vegetation (Fig. 1(d)), hard soil pavement (Fig. 1(e)) and a cement pavement (Fig. 1(f)).

In addition, raindrops, dewdrops, dust and other environmental blemishes would cause some image spots to make segmentation, location and identification more difficult compared to the industrial fields. Therefore, it is necessary to select a reasonable colour model to represent and extract the colour features of the visual images [11], [12].

\subsection{Colour Feature Analysis}

The image collected by the camera is stored in the RGB format, which is suitable for "Truecolor" display system, but it is not suitable for image segmentation and analysis. To realize the colour image segmentation, it is necessary to select an appropriate colour model and use a proper segmentation method.

The appropriate colour space can enhance the adaptability and instantaneity of the visual system, which is one of key steps of image segmentation. At present, the colour space models widely researched and used are HSI, normalized RGB and CIE. HSI colour space is defined by the components of $H, S$ and $I$, which are the hue, saturation and intensity, respectively. The $I$, intensity component, is mainly affected by illumination and view angle, and $H$ and $S$ are determined by the characteristics of the light absorption and reflection of the raw materials [13]. HSI colour space nicely fits people's colour perception. In the scene of shadows or uneven lightness, because the hue has nothing to do with the lightness and shadows, the $H$ component can effectively distinguish different colour objects,

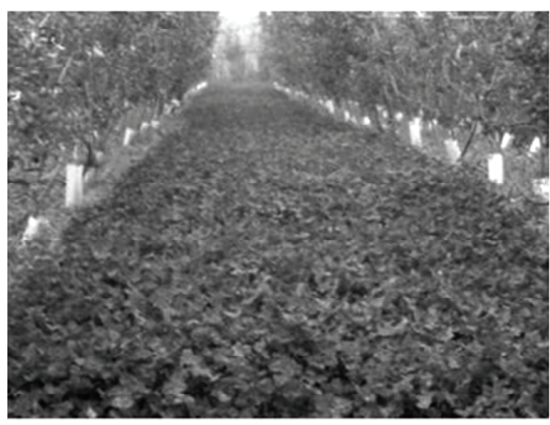

(a)

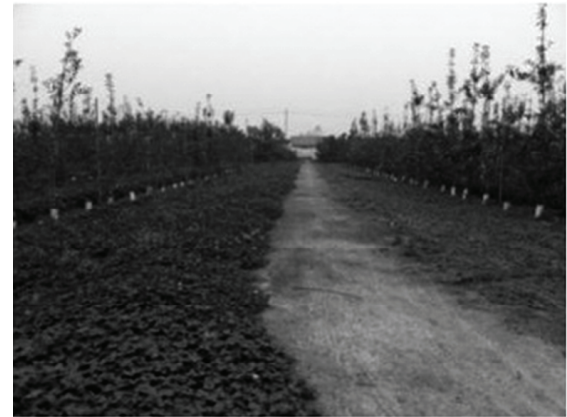

(d)

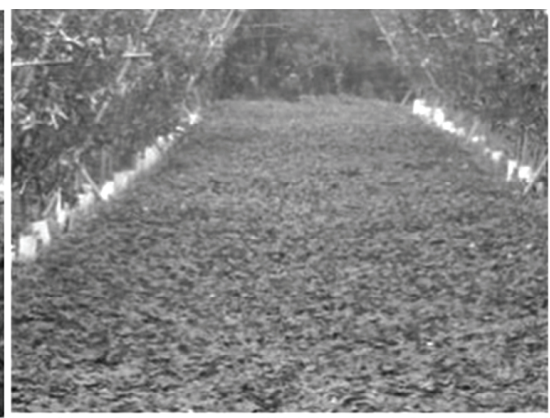

(b)

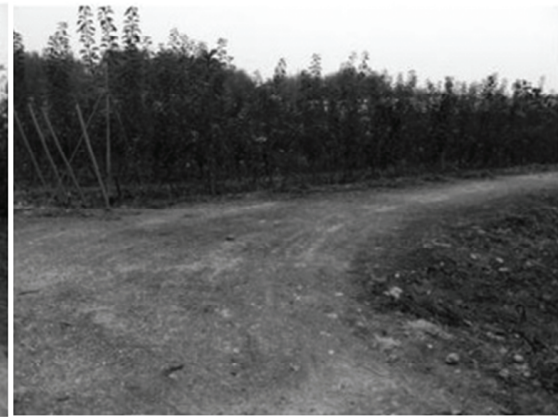

(c)

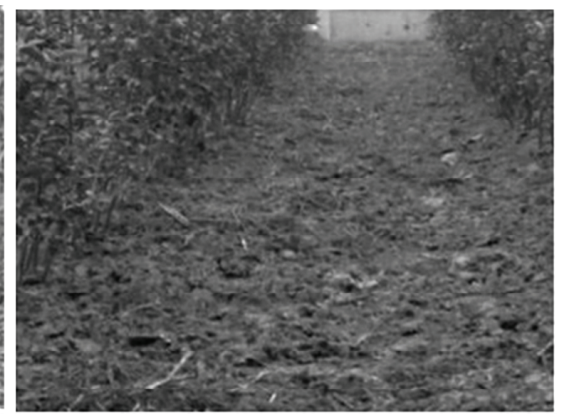

(c)

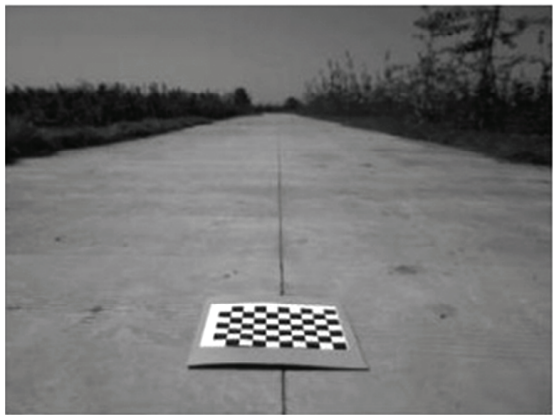

(f)

Figure 1. Apple orchard images with different field conditions. The image (a) was planted grass between two rows of apple trees and taken under $300 \mathrm{Lx}$; (b) was taken under 1,600 Lx; (c) was little-cultivated soil; (d) was part vegetation; (e) was hard soil; and (f) was cement pavement taken by a camera of Logitech QuickCam. The light intensity was measured by TES-1335 digital light meter. 
which is widely used in the target recognition applications under the complex background. Although the normalized RGB reduces the effect of image brightness on colours, it is sensitive to noise in lower luminance conditions. CIE uniform colour space $\left(L^{*} a^{*} b^{*}\right.$ and $\left.L^{*} u^{*} v^{*}\right)$ compares different colours using the geometric distance of the colour space, which can effectively measure the small colour difference. LAB colour space is the most uniform colour space, which has nothing to do with the equipment, and it is suitable for any near-natural lighting occasions.

$L^{*} a^{*} b^{*}$ colour space is based on hue and brightness, which is applicable to the representation and computation of all light sources or objects colours. Among them, $L^{*}$ denotes the brightness which value range is from 0 to $100, a^{*}$ represents the green-red component which value range is from -128 to +127 and $b^{*}$ is the blue-yellow component which value range is from -128 to +127 . $L^{*} a^{*} b^{*}$ model is often used for the detection model of the fruit harvesting robot. Huang et al. used the both ends of a channel in $L^{*} a^{*} b^{*}$ colour space just to represent the ripe tomato fruit colours and the background colours, and segmented the tomato images based on the respective colour channel [14]. Xie et al. used a channel of the Lab colour model to segment the ripe strawberry images. After the strawberry contour information was extracted, according to the mathematical model of the strawberry contour, the Hough transform was applied to recognize the ripe strawberries [15].

$L^{*} a^{*} b^{*}$ colour model can better distinguish the target and background of a colour image, and the algorithm is simple, real-time and robust. Therefore, the $L^{*} a^{*} b^{*}$ model is used to segment the robot's walking area from the background, which can improve the system's speed and efficiency.

\subsection{Apple Orchard Image Processing Based on $\boldsymbol{L}^{*} \boldsymbol{a}^{*} \boldsymbol{b}^{*}$ Model}

In this paper, the $L^{*} a^{*} b^{*}$ colour model is used to detect the robot's walking area, and the one-dimensional fuzzy entropy is used to segment the image. After the residue removal of segmented image, the image coordinate of the obstacle is obtained.
First, the RGB image is converted to the absolute colour space $X Y Z$ by [16]:

$$
\left(\begin{array}{l}
X \\
Y \\
Z
\end{array}\right)=\left(\begin{array}{lll}
0.4124 & 0.3576 & 0.1805 \\
0.2426 & 0.7152 & 0.0722 \\
0.0193 & 0.1192 & 0.9505
\end{array}\right)\left(\begin{array}{l}
\mathrm{R}_{\text {linear }} \\
\mathrm{G}_{\text {linear }} \\
\mathrm{B}_{\text {linear }}
\end{array}\right)
$$

The $X Y Z$ is converted to CIE $L^{*} a^{*} b^{*}$ (CIELAB) by:

$$
\begin{aligned}
& L^{*}=116 f\left(Y / Y_{n}\right)-16 \\
& a^{*}=500\left[f\left(X / X_{n}\right)-f\left(Y / Y_{n}\right)\right] \\
& b^{*}=200\left[f\left(Y / Y_{n}\right)-f\left(Z / Z_{n}\right)\right]
\end{aligned}
$$

where $f(t)$ is defined as:

$$
f(t)= \begin{cases}t^{1 / 3} & t>(6 / 29)^{3} \\ \frac{1}{3}\left(\frac{29}{6}\right)^{2} t+\frac{4}{29} & \text { others }\end{cases}
$$

where $X_{n}, Y_{n}$ and $Z_{n}$ are $0.950,1$ and 1.089 , respectively, according to CIE $X Y Z$ three colour stimulus values referring to the white spot.

Then, filter out the green background by [17]:

$$
a^{*}=\left\{\begin{array}{ll}
a^{*} & a^{*}>T \\
T & a^{*} \leq T
\end{array} \quad T=-5.8\right.
$$

where $T$ is a constant.

Based on the $L^{*} a^{*} b^{*}$ colour model, the results of the apple orchard images are shown in Figs. 2 and 3. Figure 2(a) is the original grass pavement image. Figure $2(\mathrm{~b})$ is the $a^{*}$ grey image, the robot walking green region is substantially filtered out, and the obstacle areas are effectively preserved. Figure 2(c) is the grey histogram of $a^{*}$ component, a large number of grey values are concentrated in the portion of low grey values. Figure $3(\mathrm{a})$ is the original cement pavement image. Figure $3(\mathrm{~b})$ is the grey image of $a^{*}$ component, which the green background and the blue

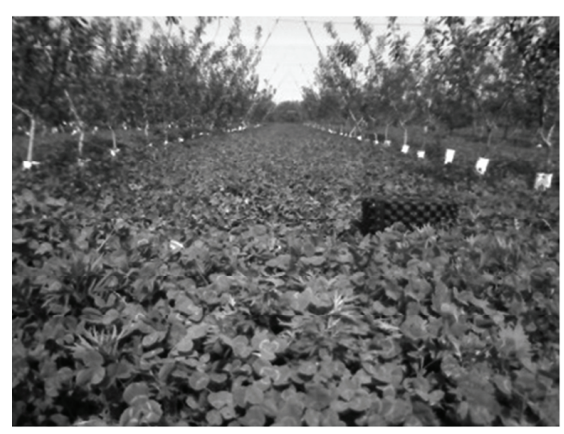

(a)

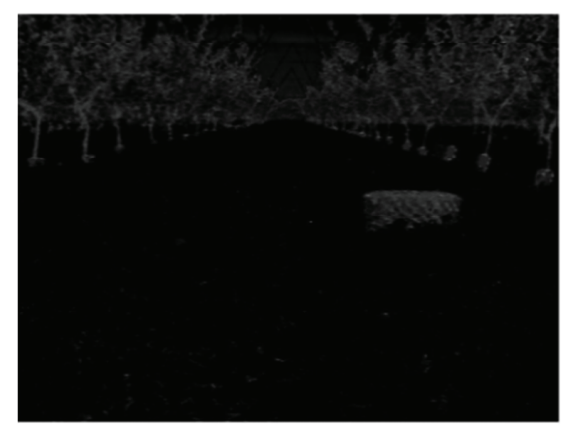

(b)

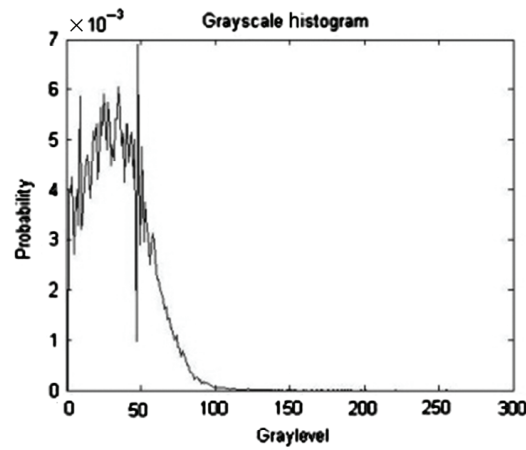

(c)

Figure 2. Processing results of grass pavement image based on $L^{*} a^{*} b^{*}$. The (a) is the original image; (b) is the grey image of $a^{*}$ component; and (c) is the grey histogram. 


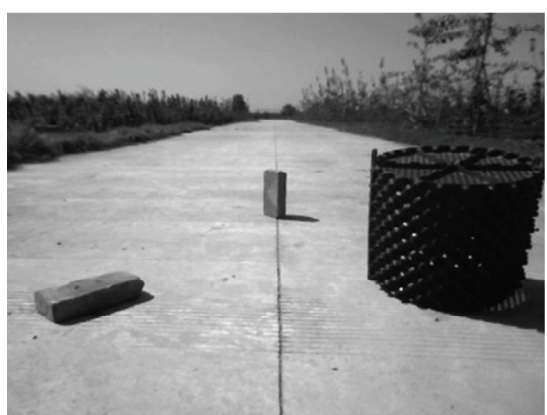

(a)

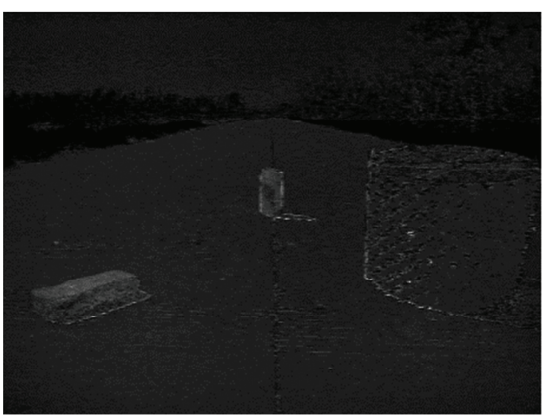

(b)

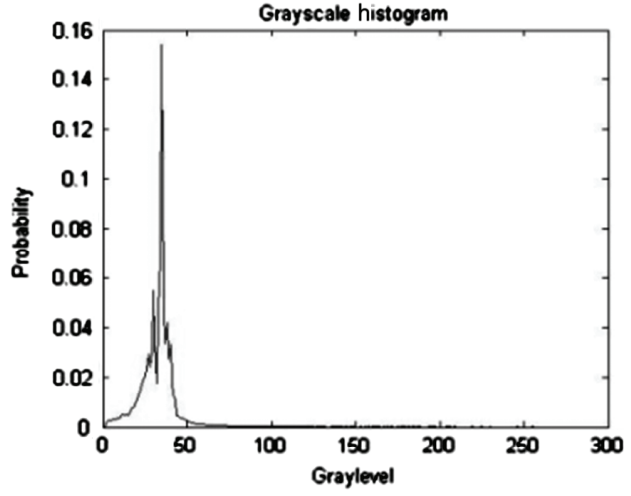

(c)

Figure 3. Processing results of cement pavement image based on $L^{*} a^{*} b^{*}$. The (a) is the original image; (b) is the grey image of $a^{*}$ component; and (c) is the grey histogram.

sky become black, and the obstacle part turns white. The obstacle region is effectively preserved. Figure $3(\mathrm{c})$ is the single peak distribution histogram of $a^{*}$ grey image, which the main grey value is concentrated in the low grey values. As can be seen above, using the $a^{*}$ component of $L^{*} a^{*} b^{*}$ colour space, the regions between two rows of apple trees and the background can be significantly distinguished, and the region that robot can walk can be effectively segmented out.

\subsection{Image Segmentation Based on One-dimensional Fuzzy Entropy}

After one apple orchard image of the natural environment is processed by the $L^{*} a^{*} b^{*}$ model, the grey values are usually concentrated in a certain range values, and its distribution is not regular shown in Fig. 2(c). In this case, the conventional image segmentation methods, such as Watershed and Otsu, are not suitable for orchard image segmentation in natural environment. Due to the complexity, the corresponding improved methods are difficult to meet the real-time requirements [18]. Therefore, this paper uses the fuzzy method to segment image, which has high efficiency and good segmentation effect.

Fuzzy entropy describes the fuzzy degree of image segmentation. Assuming a size of $M \times N$ pixels grey image, we let $x_{m n}$ is the image's grey value at the pixel coordinates $(m, n)$. A represents a fuzzy set or a fuzzy event, which can be a target or background. $\mu_{A}\left(x_{m n}\right)$ represents the fuzzy membership function of the set $\mathrm{A}$, that is level value which the pixel of the grey value $x_{m n}$ belongs to the fuzzy set A.

If the probability of the fuzzy set $\mathrm{A}$ is $P(A)$ [19]:

$$
P(A)=\frac{1}{M N} \sum_{m=1}^{M} \sum_{n=1}^{N} \mu_{A}\left(x_{m n}\right)
$$

For the threshold segmentation of the grey image, it refers to using a certain grey threshold to divide the image into two categories of the target and the background. Assumed by $A_{l}$ to represent the background, and $A_{2}$ to indicate the target, the fuzzy entropy of the image is $H$, which is defined as:

$$
H=-P\left(A_{1}\right) \log P\left(A_{1}\right)-P\left(A_{2}\right) \log P\left(A_{2}\right)
$$

When $P\left(A_{1}\right)=P\left(A_{2}\right)=\frac{1}{2}, H$ reaches the maximum value. That is to say, when both of the probabilities are equal, the entropy reaches the maximum value.

Using the maximum value of the fuzzy entropy $H$ as the separation threshold, this can retain as much information as possible in the original image after the segmentation. Using the threshold segmentation, the image can be divided into two fuzzy sets of the target (bright) and the background (dark), which membership function can be defined as:

$$
\begin{gathered}
\mu_{\text {bright }}\left(x_{m n}\right)=\left\{\begin{array}{cc}
0 & x_{m n} \leq a \\
\frac{x_{m n}-a}{c-a} & a<x_{m n}<c \\
1 & x_{m n} \geq c
\end{array}\right. \\
\mu_{\text {dark }}\left(x_{m n}\right)=\left\{\begin{array}{cc}
1 & x_{m n} \leq a \\
\frac{x_{m n}-c}{a-c} & a<x_{m n}<c \\
0 & x_{m n} \geq c
\end{array}\right.
\end{gathered}
$$

where $[a, c]$ is the fuzzy interval; $[0, a]$ and $[c, L-1]$ are the non-fuzzy interval; $\mu_{\text {dark }}\left(x_{m n}\right)$ is the degree which the pixel $(m, n)$ belongs to the background $\mu_{\text {bright }}\left(x_{m n}\right)$ is the degree which the pixel $(m, n)$ belongs to the target, and $\mu_{\text {bright }}\left(x_{m n}\right)+\mu_{\text {dark }}\left(x_{m n}\right)=1$.

When $b$, the segmentation threshold, is equal to $\frac{a+c}{2}$, the degree which the pixel with the grey value of $b$ belongs to the target or the background are 0.5. The pixel with less grey value than $b$ belongs to the background; the pixel with more grey value than $b$ belongs to the target. The fuzzy entropy $H$ of the image is defined as:

$$
H=-P \text { (bright }) \log P(\text { bright })-P(\text { dark }) \log P(\text { dark })
$$


where:

$$
\begin{aligned}
P(\text { bright }) & =\frac{1}{M N} \sum_{m=1}^{M} \sum_{n=1}^{N} \mu_{\text {bright }}\left(x_{m n}\right) \\
P(\text { dark }) & =\frac{1}{M N} \sum_{m=1}^{M} \sum_{n=1}^{N} \mu_{\text {dark }}\left(x_{m n}\right)
\end{aligned}
$$

Because of $P($ bright $)+P($ dark $)=1$, then:

$$
\begin{aligned}
H= & -P(\text { bright }) \log P(\text { bright }) \\
& -(1-P(\text { bright })) \log (1-P(\text { bright }))
\end{aligned}
$$

According to the information theory, the greater entropy of a probability event contains the greater amount of information. If we want to achieve the ideal segmentation of the target and the background, the entropy $H$ of the image should be the maximum value. Therefore, the optimal fuzzy parameters $a_{\text {opt }}$ and $c_{\text {opt }}$ can be obtained when the $H$ is the largest value, then we can get the optimal segmentation threshold $b_{\mathrm{opt}}=\left(a_{\mathrm{opt}}+c_{\mathrm{opt}}\right) / 2$.

If using exhaustive method to search the maximum one-dimensional entropy, the algorithm is too complex to meet the system's real-time requirement. This paper adopts the improved exhaustive search method to search the maximum entropy. The pseudo code for the improved exhaustive searching method is as follows:

The pseudo code for the improved exhaustive searching method

1. Input the grey image;

2. Calculate the probability of each grey $x_{m n}$, and find the grey Hist max $_{\text {of maximum }}$ probability;

3. for (a in [Hist max $\left._{254]}\right)$

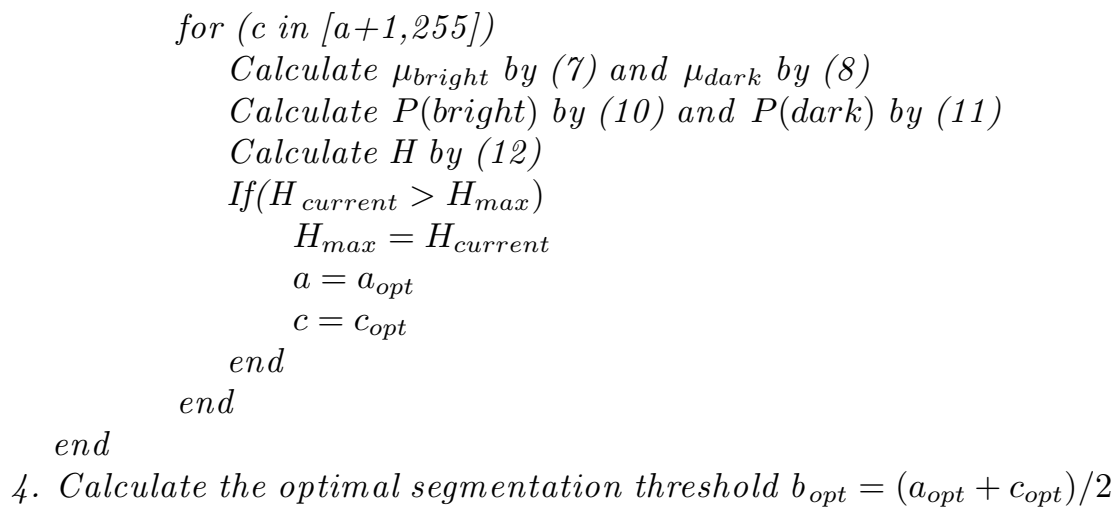

For the grey image processed by $L^{*} a^{*} b^{*}$ model, we use the one-dimensional fuzzy entropy to segment the image, use the method of mathematical morphology to remove residues, and use the positive order $3 \times 3$ structural elements to conduct the square root operation to filter out the segmentation fragments of size of $1 \% \times 1 \%$. For Fig. 2(b), the results are shown in Fig. 4, which the obstacles are effectively retained and the effect is obvious when the optimal threshold is 33 and the maximum entropy is 0.8064 . For Fig. 3(b), the results are shown in Fig. 5, which intermingle with significant noise of the cement pavement. After the processing of the mathematical morphology, the

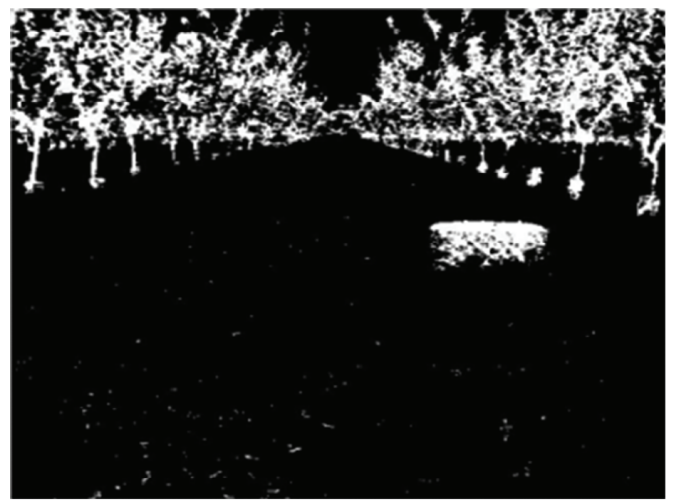

(a)

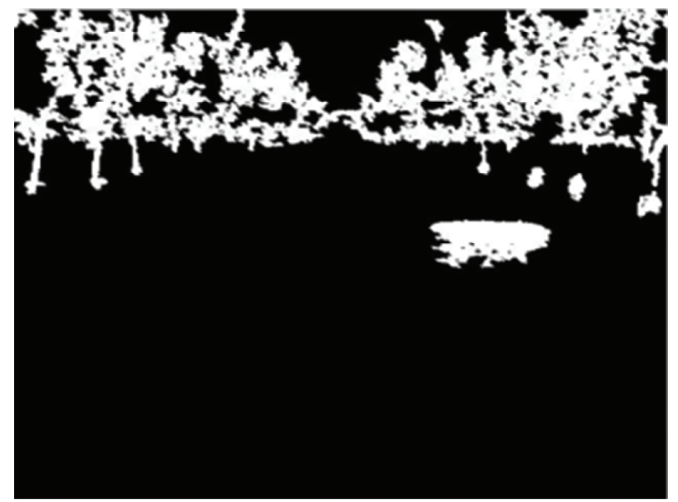

(b)

Figure 4. Segmentation results of Fig. 2(b). The image (a) is the segmentation effect when the threshold is 33 ; and (b) is the residues removal effect. 


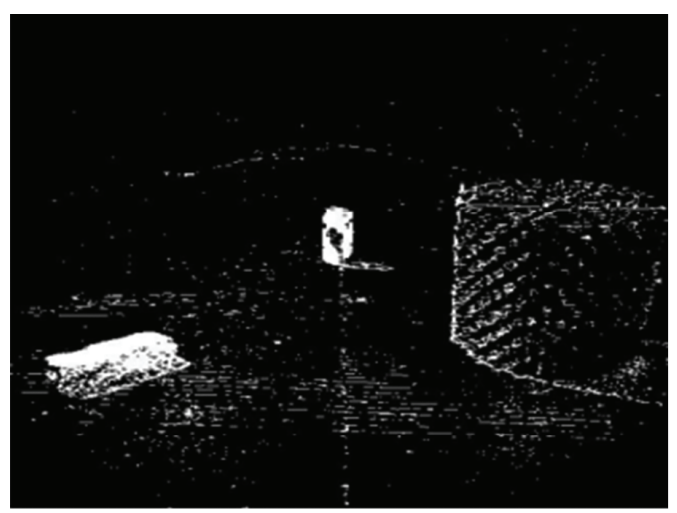

(a)

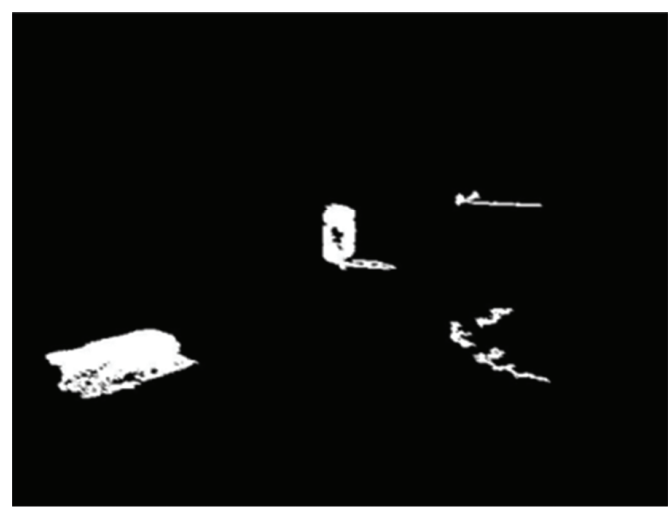

(b)

Figure 5. Segmentation results of Fig. 3(b). The image (a) is the segmentation effect when the threshold is 42 ; and (b) is the residues removal effect.

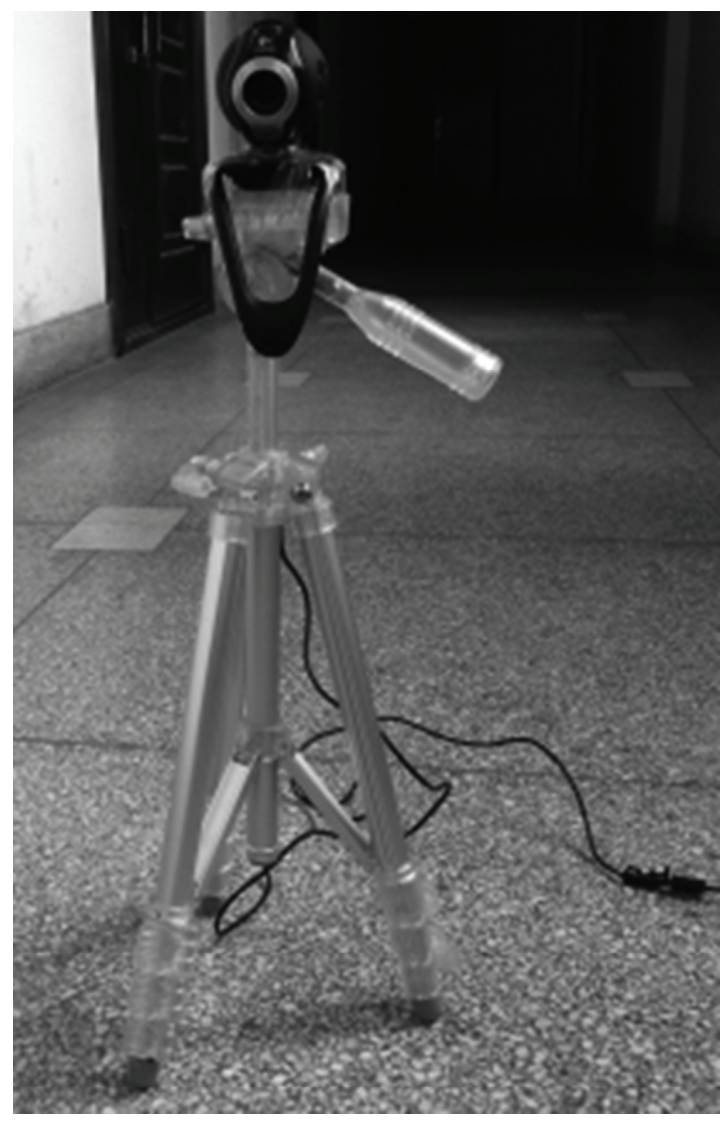

Figure 6. Image acquisition device.

residues are removed and the obstacles are effectively retained when the optimal threshold is 42 and the maximum entropy is 1.0267. Based on the segmentation results of Figs. 4 and 5, the high real-time requirement of the apple orchard image segmentation can be satisfied by using the one-dimensional fuzzy entropy.

\subsection{Inverse Perspective Transformation}

To obtain the ground information of the robot walking area, we use the inverse perspective transformation to transform the ground perspective image into a top view [20]. According to the installation requirements of the

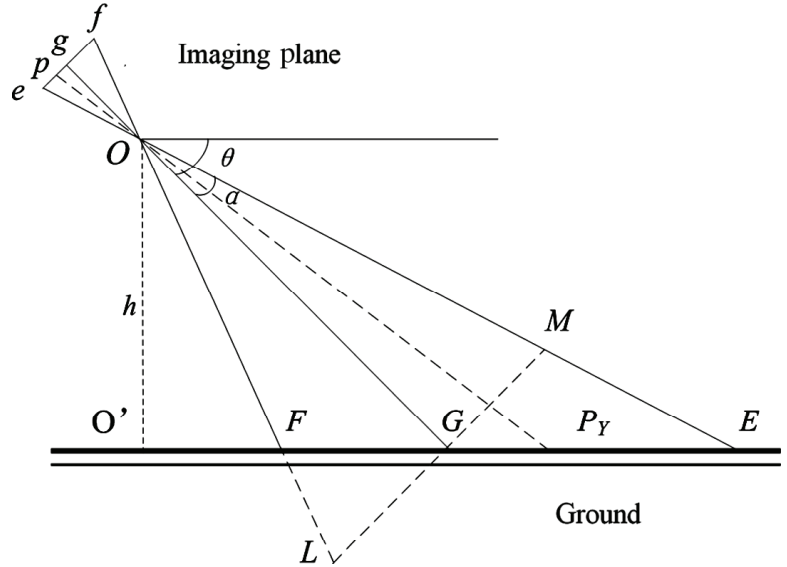

Figure 7. Lateral view of view field.

robot's camera, this study uses a common camera to build a monocular vision system. The camera is mounted on a tripod as shown in Fig. 6 . The height is adjusted to $h$, and the top view angle is adjusted to $\theta$. Figure 7 shows the lateral view of the experimental visual system, the image resolution is $m \times n$ (pixels), and the view angle is $2 \alpha \times 2 \beta$ (radians, where $2 \alpha$ is the vertical view angle).

Let a spot on the ground is $P_{Y}\left(X_{P}, Y_{P}\right)$ and the projection of the point $P$ on the imaging plane is $p(u, v)$. According to the plane geometry, the relation between the ground coordinate and the pixel coordinate can be calculated by:

$$
\left\{\begin{array}{l}
Y_{P}=h \times \cot \left(\theta-\operatorname{atan}\left(\tan \alpha \times\left(1-\frac{2 u}{m-1}\right)\right)\right) \\
X_{P}=\sqrt{h^{2}+Y_{P}^{2}} \times \frac{\tan \beta \times\left(\frac{2 v}{n-1}-1\right)}{\sqrt{1+\left(\tan \alpha \times\left(1-\frac{2 u}{m-1}\right)\right)^{2}}}
\end{array}\right.
$$

\section{Obstacle Detection}

For the processed image, the non-deleted outline between two rows of apple trees is regarded as the obstacle region. 


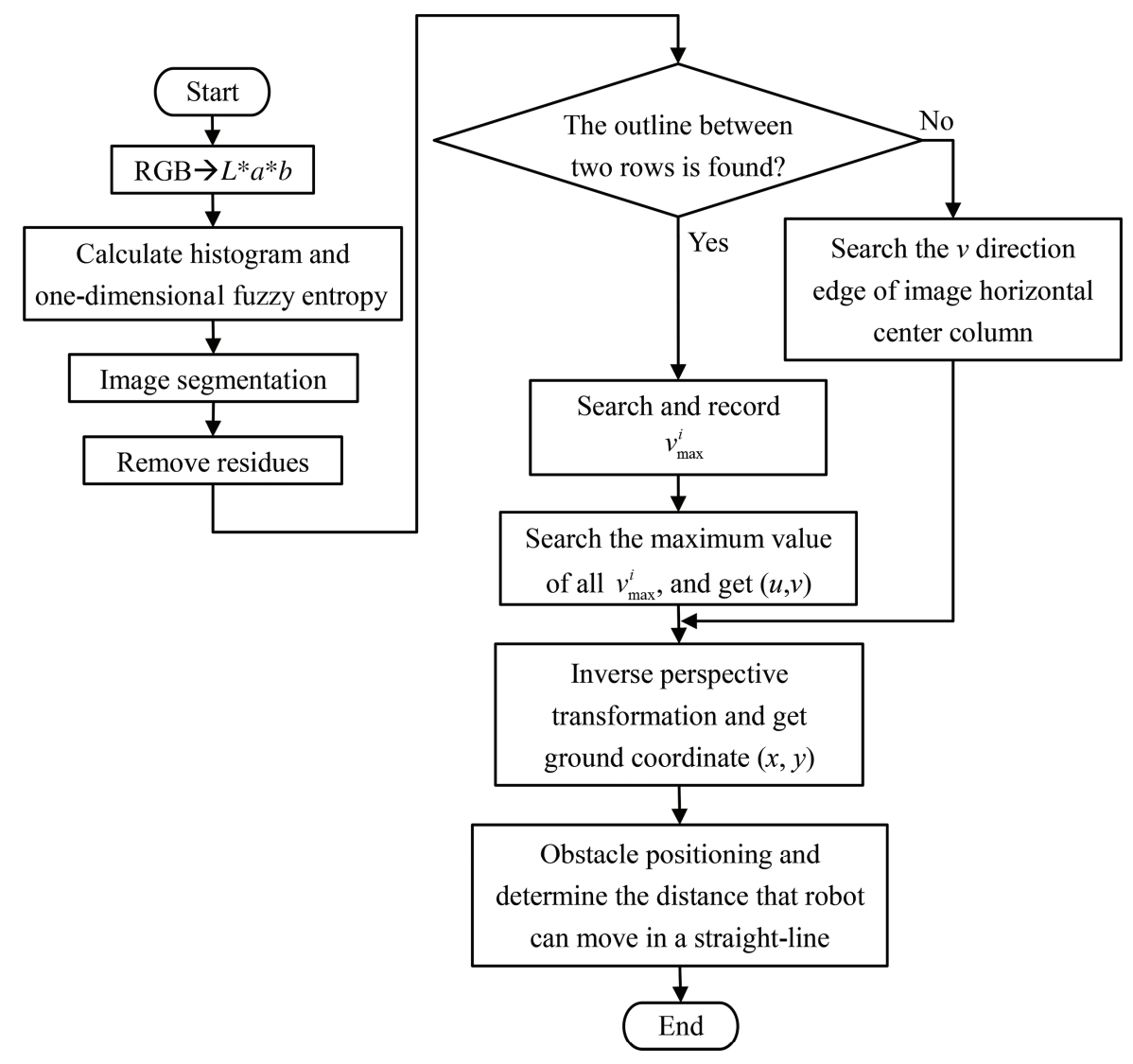

Figure 8. Flowchart of obstacle detection algorithm based on monocular vision.

For every outline, the maximum value of $v$ direction in the image coordinate system is calculated by:

$$
v_{\max }^{i}=\max \left(v_{i}\right)
$$

The maximum value of all $v_{\max }^{i}$ is calculated by:

$$
v=\max \left(v_{\max }^{i}\right)
$$

Meanwhile, the coordinate of $u$ direction is recorded, the coordinate of the point close to the robot of the obstacle is obtained. The $u, v$ coordinates are conducted in the inverse perspective transformation by (13), thus the $x, y$ coordinates of the obstacle are confirmed, which achieves the obstacle detection based on monocular vision and determines the distance that robot can move in a straight-line.

For the processed image, if the non-deleted outline between two rows of apple trees does not exist, the distance that robot can move in a straight-line is the edges of the rows. At this time, the $v$ direction edge of image horizontal centre column is defined as $v$. The coordinate $(u, v)$ is substituted in the (13) for the inverse perspective transformation, thus the distance that robot can move in a straight-line is determined. In summary, the flowchart of the obstacle detection algorithm based on monocular vision is shown in Fig. 8.

\section{Experiments and Results Analysis}

To verify the effectiveness of the obstacle detection algorithm, we programmed and experimentally verified it.

The MATLAB code was used for programming within the Windows XP professional operating system and on a personal computer equipped with a $2.50 \mathrm{GHz}$ Intel Pentium(R) Dual-Core E5200 CPU and 1 GB RAM.

In our experiments, the images were collected from the Modern Agricultural Science and Technology Demonstration Zone in Yangling, Shaanxi, China, and the data acquisition equipment is shown in Fig. 6 . The parameters were chosen as: the camera's height $h$ was $0.51 \mathrm{~m}$; the image resolution was $640 \times 480$; the view field angle $\alpha$ was 0.144 radians, $\beta$ was 0.183 radians. And, 100 images were taken under different lightness, vegetation and texture pavement between two rows of apple trees. In Fig. 9, some bricks or barrels and other obstacles were put in the robot walking area, the marking white line was overlapped with the projection of the camera optical axis on the ground, the steel tape with division value of $1 \mathrm{~mm}$ was used to measure the coordinate $(x, y)$ of the point close to the robot of the obstacle, and the average value of 10 measurement values was taken as the measuring value of $x_{T}$ and $y_{T}$.

According to Fig. 8, the obstacle detection algorithm was implemented on the personal computer. Under various illumination, and different vegetation, texture pavement between different rows, we obtained the ground coordinate 


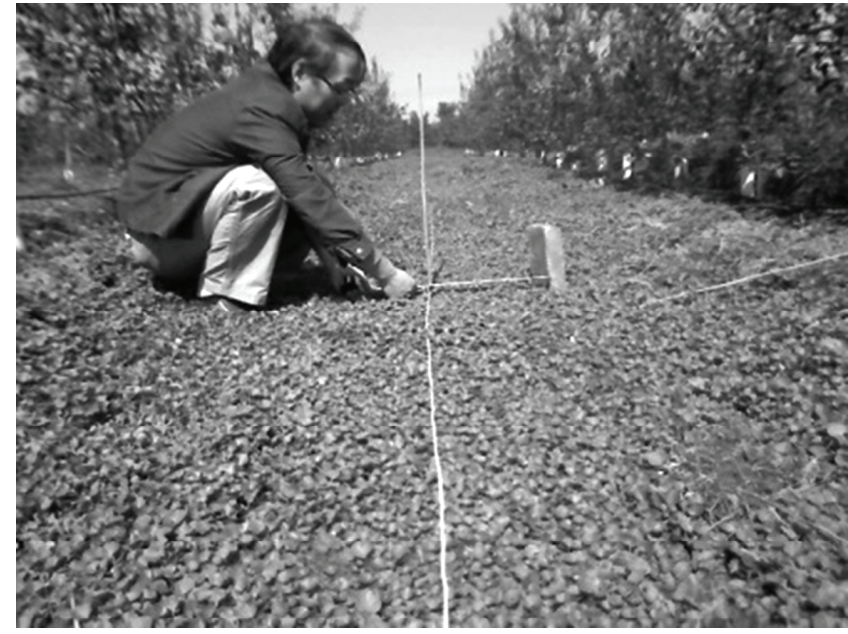

Figure 9. Field experimental images and data collecting.

of the rows' edge or the point close to the robot of the obstacle to realize the robot inline visual positioning.

The experimental results show that 92 results of 100 images are true. The correct rate is $92 \%$. The positioning results of 15 images are shown in Table 1, and the errors are shown in Table 2 correspondingly.

From the experimental results, for the $X$ coordinate of obstacles, the maximum absolute error is $4.95 \mathrm{~cm}$, and the maximum relative error is $5.75 \%$; for the $Y$ coordinate of obstacles, the maximum absolute error is $7.87 \mathrm{~cm}$, and the
Table 2

Inverse Perspective Transformation Errors of the Absolute Error $(d x, d y)$ and the Relative Error $(x, y)$

\begin{tabular}{|l|c|c|c|c|}
\hline \multirow{2}{*}{ Exp. No. } & \multicolumn{2}{|c|}{ Absolute Error } & \multicolumn{2}{c|}{ Relative Error } \\
\cline { 2 - 5 } & $d x(\mathrm{~cm})$ & $d y(\mathrm{~cm})$ & $x(\%)$ & $y(\%)$ \\
\hline 1 & 1.92 & 0.11 & 3.46 & 0.06 \\
\hline 2 & 0.19 & 1.69 & - & 0.89 \\
\hline 3 & 0.41 & 6.32 & - & 1.50 \\
\hline 4 & 1.20 & 6.58 & 4.21 & 3.27 \\
\hline 5 & 4.95 & 4.62 & - & 0.81 \\
\hline 6 & 0.52 & 2.13 & 1.45 & 1.04 \\
\hline 7 & 0.50 & 0.26 & 1.44 & 0.13 \\
\hline 8 & 1.62 & 2.45 & - & 1.34 \\
\hline 9 & 0.68 & 0.75 & 1.42 & 0.24 \\
\hline 10 & 1.08 & 1.28 & 1.86 & 1.22 \\
\hline 11 & 0.28 & 0.40 & 0.68 & 0.33 \\
\hline 12 & 0.22 & 1.85 & - & 0.80 \\
\hline 13 & 1.81 & 2.47 & 5.75 & 1.78 \\
\hline 14 & 1.38 & 7.87 & 3.34 & 6.53 \\
\hline 15 & 0.17 & 2.02 & 2.64 & 0.87 \\
\hline
\end{tabular}

Table 1

Experimental Results of 15 Images Marked by Exp. No., Including with the Coordinates $(x, y)$ of the Obstacles' Point Close to the Robot by the Inverse Perspective Transformation, the Measuring Value $\left(x_{T}, y_{T}\right)$ and the Distance $Y$, Which the Robot Can Go Straightly

\begin{tabular}{|l|r|c|r|r|r|}
\hline \multirow{2}{*}{ Exp. No. } & \multicolumn{2}{|c|}{ Obstacles' Coordinates } & \multicolumn{2}{|c|}{ Measuring Value } & \multirow{2}{*}{ Distance $Y(\mathrm{~cm})$} \\
\cline { 2 - 5 } & $x(\mathrm{~cm})$ & $y(\mathrm{~cm})$ & $x_{T}(\mathrm{~cm})$ & $y_{T}(\mathrm{~cm})$ & \\
\hline 1 & -53.58 & 199.39 & -55.50 & 199.50 & 199.39 \\
\hline 2 & 0.19 & 191.74 & 0.00 & 190.05 & 191.74 \\
\hline 3 & -0.41 & 427.47 & 0.00 & 421.15 & 427.47 \\
\hline 4 & 27.33 & 207.63 & 28.53 & 201.05 & 207.63 \\
\hline 5 & -4.95 & 574.62 & 0.00 & 570.00 & 574.62 \\
\hline 6 & 36.37 & 207.63 & 35.85 & 205.50 & 207.63 \\
\hline 7 & -35.25 & 191.74 & -34.75 & 192.00 & 191.74 \\
\hline 8 & 1.62 & 180.60 & 0.00 & 183.05 & 180.60 \\
\hline 9 & 47.52 & 311.10 & 48.20 & 311.85 & 311.10 \\
\hline 10 & -56.97 & 103.97 & -58.05 & 105.25 & 103.97 \\
\hline 11 & -40.72 & 121.45 & -41.00 & 121.05 & 121.45 \\
\hline 12 & 0.22 & 228.25 & 0.00 & 230.10 & 228.25 \\
\hline 13 & 29.74 & 136.13 & 31.55 & 138.60 & 136.13 \\
\hline 14 & 39.87 & 112.64 & 41.25 & 120.50 & 112.64 \\
\hline 15 & -6.69 & 234.57 & -6.52 & 232.55 & 234.57 \\
\hline
\end{tabular}


maximum relative error is $6.53 \%$. The obstacle detection algorithm based on monocular vision can meet the accuracy requirements of the application.

\section{Conclusion}

Based on analysing and studying the characteristics of modern apple orchard images, the apple orchard images are represented using $L^{*} a^{*} b^{*}$ colour model, then onedimensional fuzzy entropy segmentation algorithm is used for image segmentation. This paper presents simple and effective obstacles detection and walking areas extraction algorithm based on monocular vision for apple orchard robot.

The $a^{*}$ component in the $L^{*} a^{*} b^{*}$ colour space can reduce some unfavourable factors caused by variable illumination, different vegetation and texture pavement between the rows of apple trees. This can better highlight the background and the objectives, and basically solve the possible problems in natural scenes. For the detection of green or light green obstacles, other specific methods can be used or the threshold parameters can be adjusted, which needs to follow a further study.

\section{Acknowledgement}

The authors would like to thank the associate editor and anonymous reviewers for their valuable comments. This work was supported by the Natural Science Foundation Project of Shaanxi Province (Grant No. 2015JQ6246) and the major agricultural science and technology extension service project of Shaanxi Province (Grant No.2016XXPT00).

\section{References}

[1] C.T. Chao, M.H. Chung, J.S. Chiou, and C.J. Wang, A simple interface for $3 \mathrm{D}$ position estimation of a mobile robot with single camera, Sensors, 16(4), 2016, 435.

[2] S.X. Yang and C. Luo, A neural network approach to complete coverage path planning, IEEE Transactions on Systems, Man, and Cybernetics, Part B, 34(1) 2004, 718-725.

[3] C. Luo and S.X. Yang, A bioinspired neural network for realtime concurrent map building and complete coverage robot navigation in unknown environments, IEEE Transactions on Neural Networks, 19(7), 2008, 1279-1298.

[4] A. Bechar and C. Vigneault, Agricultural robots for field operations: Concepts and components, Biosystems Engineering, 149, 2016, 94-111.

[5] X. Zhao, J.Z. Yuan, and H.Z. Liu, Advances in vision-based target location technology, Computer Science, 43(6), 2016, 10-16.

[6] M. Perrollaz, R. Labayrade, D. Gruyer, A. Lambert, and D. Aubert, Proposition of generic validation criteria using stereo-vision for on-road obstacle detection, International Journal of Robotics and Automation, 29(1), 2014, 32-43.

[7] X. Li, C. Luo, Jean Dezert, and Y. Tan, Generic object recognition based on feature fusion in robot perception, International Journal of Robotics and Automation, 31(5), 2016, DOI: 10.2316/Journal.206.2016.5.206-4706.

[8] L. Zhang, S.M. Wang, B.Q. Chen, and Z.G Liu, Detection of obstacles in farmland based on binocular vision, Journal of China Agricultural University, 12(4), 2007, 70-74.

[9] G.L. Li, C.Y. Ji, and B.X. Gu, Recognition and location of oscillating fruit based on monocular vision and ultrasonic testing, Transactions of the Chinese Society of Agricultural Machinery, 46(11), 2015, 1-8.

[10] S.W. Jia, J.M. Li, Q. Qiu, and H.J. Tang, New corridor edge detection and navigation for greenhouse mobile robots based on laser scanner, Transactions of the Chinese Society of Agricultural Engineering, 31(13), 2015, 39-45.

[11] L.W. Huang, D.J. He, and S.X. Yang, Segmentation on ripe Fuji apple with fuzzy 2D entropy based on 2D histogram and GA optimization, Intelligent Automation and Soft Computing, 19(3), 2013, 239-251.

[12] J.F. Zhang, D.J. He, and L.W. Huang, Recognition and segmentation of Fuji apples in orchards based on 2D entropy, International Journal of Digital Content Technology and Its Applications, 6(18), 2012, 572-578.

[13] X.M. Pang, Z.J. Min, and J.M. Kan, Color image segmentation based on HSI and LAB color space, Journal of Guangxi University, 36(6), 2011, 976-980.

[14] L.W. Huang, S.X. Yang, and D.J. He, Abscission point extraction for ripe tomato harvesting robots, Intelligent Automation and Soft Computing, 18(6), 2012, 751-763.

[15] Z.Y. Xie, T.Z. Zhang, and J.Y. Zhao, Ripened strawberry recognition based on Hough transform, Transactions of the Chinese Society for Agricultural Machinery, 38(3), 2007, 106109.

[16] H. Yin, Y. Chai, S.X. Yang, and G.S. Mittal, Ripe tomato extraction for a harvesting robotic system, Proceedings of the 2009 IEEE International Conference on Systems, Man and Cybernetics. San Antonio, TX, USA, 2009, 2984-2989.

[17] W.M. Syahrir, A. Suryanti, and C. Connsynn, Color grading in tomato maturity estimator using image processing technique, 2009 2nd IEEE International Conference on Computer Science and Information Technology (ICCSIT 2009), Beijing, China, 2009, 276-280.

[18] Y.X. Wang, S.X. Sun, and J.D. Zhong, An ensemble anomaly detection with imbalanced data based on robot vision, Inter national Journal of Robotics and Automation, 31(2), 2016, 77-83.

[19] H.D. Cheng and H.J. Xu, A novel fuzzy logic approach to contrast enhancement, Pattern Recognition, 33(5), 2000, 809-819.

[20] Y. Cao, Y. Feng, Y.T. Yang, Y.J. Chen, B. Lei, and L.S. Zhao, Monocular visual odometry based on inverse perspective mapping, International Symposium on Photoelectronic Detection and Imaging 2011: Advances in Imaging Detectors and Applications, Beijing, China, 2011, DOI: 10.1117/12.900010.

\section{Biographies}

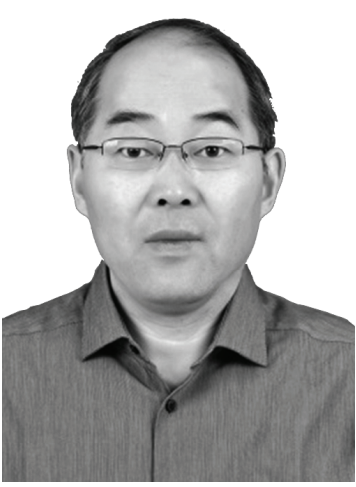

Jianfeng Zhang received his Ph.D. degree in the College of Mechanical and Electric Engineering, Northwest A\&F University, Yangling, Shaanxi, China, in 2012. His research interests mainly focus on the key technologies of intelligent control system and applications, motion control of robotic systems and embedded systems in the application of modern agriculture.

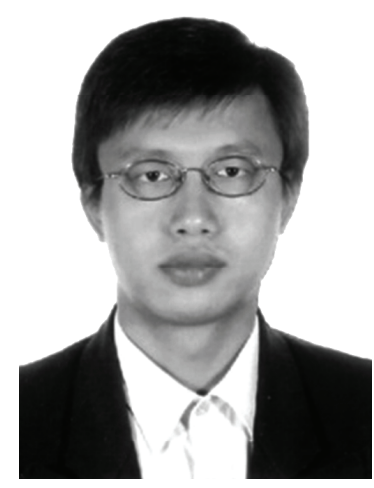

Bin Yang received his Ph.D. degree in the School of Electronic and Information Engineering, Xi'an Jiaotong University, Xi'an, Shaanxi, China, in 2014. His research interests mainly focus on the physical layer security, signal processing in wireless communication system and embedded systems in the application of modern agriculture. 


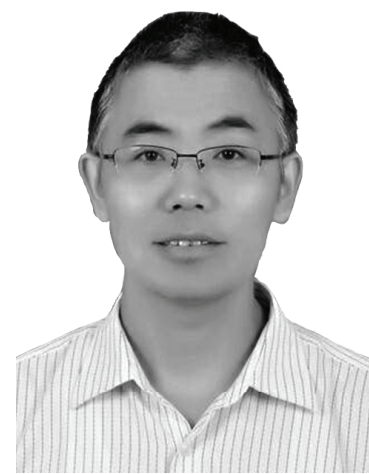

Nan Geng received his Ph.D. degree in the College of Mechanical and Electric Engineering, Northwest A\&F University, Yangling, Shaanxi, China, in 2012. His research interests on image analysis and machine vision, computer virtual technology and graphics, and agricultural informatization. $\mathrm{He}$ is a professor of College of Information Engineering, Northwest A\&F University and Director of the Institute of Graphic Image at ShaanXi province.

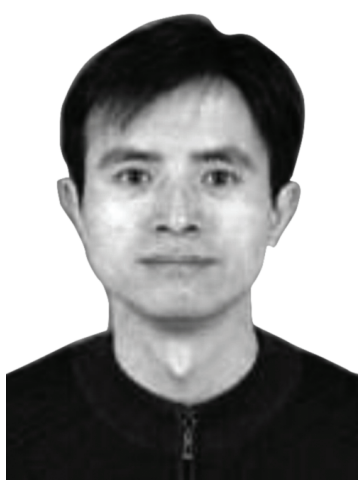

Lvwen Huang received his Ph.D. degree in agriculture electrification and automation from College of Mechanical and Electric Engineering, Northwest A\&F University, Yangling, Shaanxi, China, in 2013. His research interests lie in intelligent system and image processing. 